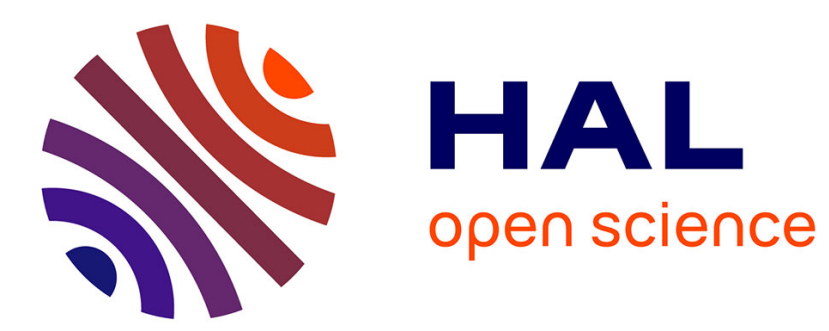

\title{
THE CONDENSED MATTER PHYSICS OF PLANETARY INTERIORS
}

\author{
D. Stevenson
}

\section{To cite this version:}

D. Stevenson. THE CONDENSED MATTER PHYSICS OF PLANETARY INTERIORS. Journal de Physique Colloques, 1980, 41 (C2), pp.C2-53-C2-59. 10.1051/jphyscol:1980208 . jpa-00219800

\section{HAL Id: jpa-00219800 https://hal.science/jpa-00219800}

Submitted on 1 Jan 1980

HAL is a multi-disciplinary open access archive for the deposit and dissemination of scientific research documents, whether they are published or not. The documents may come from teaching and research institutions in France or abroad, or from public or private research centers.
L'archive ouverte pluridisciplinaire HAL, est destinée au dépôt et à la diffusion de documents scientifiques de niveau recherche, publiés ou non, émanant des établissements d'enseignement et de recherche français ou étrangers, des laboratoires publics ou privés. 


\title{
THE CONDENSED MATTER PHYSICS OF PLANETARY INTERIORS
}

\author{
D. J. Stevenson
}

Department of Earth \& Space Sciences, University of Califormia - Los Angeles, CA 90024

Résumé.- Les intêrieurs profonds de Jupiter et Saturne se composent de l'hyđrogène fluide et métallique. A l'intérieur de Jupiter, l'hyarogène métallique et l'hélium sont mélangés uniformément. A l'intérieur de Saturne, la solubilité incomplète de l'hélium fait nastre la dissolution et la libération d'énergie. Aux intérieurs de Neptune et Uranie, le mellange fluide (de l'hydrogẽne, de l'hélium, de l'eau, du méthane et de l'amoniaque) est métallique ou ionisé. Aux centres terrestres, le fer métallique prédomine.

Abstract.- The deep interiors of Jupiter and Saturn consist of fluid, metallic hydrogen. In Jupiter the metaliic hydrogen is uniformly mixed with helium. In Saturn, the incomplete solubility of helium may cause unmixing and energy release. In the interiors of Uranus and Neptune the fluid mixture (of hydrogen, helium, water, methane and ammonia) is metallic or ionic. The terrestral planetary cores are predominantly metallic iron.

1. Introduction. - The cosmic abundances of the elements are reflected in the compositions of the planets in our solar system. The terrestrial planets contain a very biased sample of cosmic matter, consisting almost entirely of refractory elements ("rock"). Most of the planetary mass has a composition similar to that of the primordial sun and resides in the two gas giants, Jupiter and Saturn. This short review will adopt a cosmic (non-anthropocentric) view, which necessarily reduces the emphasis on the materials which we have most experience with: silicates and iron. Planetary modellers need to understand the behavior of matter over the entire range of conditions from an ideal gas to a degenerate Coulomb plasma. Degeneracy in the deep interiors of planets is assured (this is the distinction between stars and planets; even Jupiter is a factor of sixty less massive than the lowest mass main sequence star). However, the degree of pressure-ionization depends on the mass of the planet, since the mass determines the gravitational force and hence (by the equation of hydrostatic equilibrium) the pressure difference between surface and center. A characteristic internal pressure $\bar{P}$ is $\bar{\rho} g R$, where $\bar{\rho}$ is the average planetary density, $g$ is the gravitational acceleration and $R$ is the planetary radius. This can be conveniently expressed as

$\overline{\mathrm{P}} \simeq 2.5\left(\frac{\mathrm{M}}{\mathrm{M}_{\oplus}}\right)^{2 / 3}\left(\frac{\bar{\rho}}{\bar{\rho} \oplus}\right)^{4 / 3} \times 10^{11} \mathrm{~Pa}$

Where $M$ is the planetary mass and the symbol $\oplus$ refers to the earth. Typical interior pressures are thus $2.5 \times 10^{11} \mathrm{~Pa}$ in the earth and $2 \times 10^{12} \mathrm{~Pa}$ in Jupiter. It is of interest to compare this with the pressure required for metallizing the elements. of the ten most abundant elements (by mass) in the universe, only iron is commonly found in its free metallic form. Of the rest, only magnesium is a metal in its elemental form, but magnesium is invariably combined with silicon and oxygen under the conditions prevailing during and after planetary formation. Iron is also rather insoluble in magnesium silicate; it is not therefore surprising that the terrestrial planets appear to have predominantly iron cores.

The metallization of elements or compounds which are "normally" insulating occurs when the work done by pressure on the material is comparable to $I$, the characteristic electronic energy difference between insulating and conducting states:

$P_{\operatorname{met}^{2 I / V}}$

where $\mathrm{V}$ is the volume per atom or molecule (as appropriate). This is only an order of magnitude estimate, but for I few electron volts and $\mathrm{v}^{\prime} 10 \mathrm{a}_{0}^{3}\left(a_{0}\right.$ is the first Bohr radius) the metallization pressure

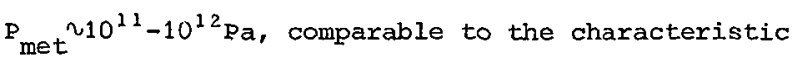
internal pressures of bodies that are at least as massive as the earth. We might expect, therefore, to find that the deep interiors of all planets at least as massive as the earth are metallic, regard less of whether iron is present. It has even been suggested that the metaliic state of the earth's core is a consequence of the metallization of silicates (Ramsey, 1949 ; Lyttleton, 1973 ) but this cannot be readily reconciled with the seismic data and shock wave data (Birch, 1968). To proceed further, we need to identify more precisely the compositions of the planets. As a first 
approximation, we can assume zero temperature (because the thermal energy of a planet is much less than the gravitational energy) and estimate the equation of state of the elements using Thomas-Fermi-Dirac theory. The results of such a calculation by zapolsky and Salpeter (1969) are shown in figure 1 .

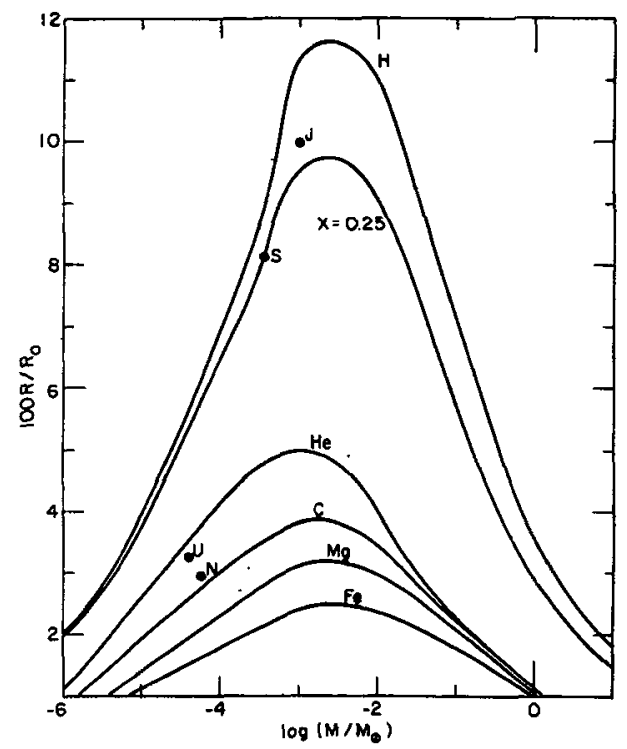

Fig. I : Mass-radius relationship (in solar units) for zero temperature spheres of various compositions. $\mathrm{X}=0.25$ labels a $75 \% \mathrm{H}-25 \% \mathrm{He}$ mixture. J, S,U,N represent the giant planets.

This figure illustrates several important points. First, we see that in low mass objects $\mathrm{R}^{\circ M^{1 / 3}}$ approximately applies, because the internal pressure is less than the bulk modulus of the materials, so the density is close to the zero-pressure density. At high masses, however, the balance is between gravity and the Fermi degeneracy pressure of the electrons. In this limit (the domain of white dwarf) $\mathrm{R}^{\mathrm{M}} \mathrm{M}^{-1 / 3}$. Jupiter is intermediate and is within $20 \%$ in radius of being the largest cold body in the universe (although, of course, there are probably an enormous number of similar bodies).

Second, we can see from Fig. 1 that Jupiter and Saturn are predominantly hydrogen if they are cold. (We shall see below that the assumption of a "cold" body is a good first approximation). In fact, they can be modelled by an approximately cosmic mixture of 75 hydrogen, $25 \&$ helium. The figure shows that there is a very large difference between the massradius relationship of predominantly hydrogen bodies and the relationship for any other element. The attribution of a predominantly hydrogen composition to Jupiter and saturn is therefore unambiguous. Third, the figure suggests that Uranus and Neptune are not predominantly hydrogen and helinum but are enriched in the ice-forming elements $(O, N, C)$ which occur as water, ammonia and methane in a hydrogen-rich environment. In fact, the compositions of Uranus and Neptune are ambiguous, but current models invoke roughly comparable amounts of rock, ice and gas (Hubbard and MacFarlane, 1979). The terrestrial planets are not shown on Fig. 1 since they are outside the domain of applicability of Thomas-Fermi-Dirac theory. To a first approximation, the earth, Venus and Mars are each about onethird metallic iron and two-thirds magnesium-iron silicates by mass. Mercury is enxiched in iron (about three quarters of its mass is in an iron core), whilst the moon is depleted in iron (a very small iron core, if any).

The internal temperatures of planets are less readily deduced than the composition. In all the planets, the internal heat flux (caused primarily by radioactivity in the terrestrial planets and pximarily by the gradual loss of primordial heat in the major planets) is greater than that which can be transported along an adiabat by conduction. It follows that convection is the dominant form of heat transport almost everywhere in all bodies larger than about 1000 kilometers in radius. The internal temperature prodile will be approximatley adiabatic, but the choice of adabat is determined by the atmospheric properties in the major planets, whereas it is determined by the rheology of silicates in the terrestrial planets. In Jupiter, for example, the temperature profile is approximately an adiabat at all pressures exceeding about $10^{5} \mathrm{~Pa}$, and can be crudely represented by

$T \sim 10,000 p^{1 / 2} 0_{K}$

where $\rho$ is the density in $\mathrm{g} \cdot \mathrm{cm}^{-3}$. The assumption of adiabaticity may be violated if there is a phase transition. Nevertheless, internal temperatures of order $10,000^{\circ} \mathrm{K}$ characterize Jupiter and Saturn; whereas temperatures of order $3000^{\circ} \mathrm{K}$ are more typical of Uranus, Neptune and the terrestrial planets. In all cases, the product $\alpha T$, where $\alpha$ is the coefficient of thermal expansion, is less than 0.05 , 
and this justifies our assertion that planets are "cold".

2. Hydrogen. - At very high pressures, hydrogen must become a Coulomb plasma: protons immersed in an almost uniform degenerate sea of electrons. This state, known as metallic hydrogen, is in most respects the simplest of the alkali metals, and its equation of state can be calculated accurately from first pxinciples. Apart from screening corrections, metallic hydrogen is the standard and extensively studied one component plasma (Pollock and Hansen, 1973). The classical one component plasma melts at around $\Gamma_{\simeq 155}$ where $\Gamma \equiv e^{2} /\left(k_{B} \operatorname{Tr} s_{o}{ }_{o}\right)$ is the usual plasma parameter (e is the electron charge, $k_{B}$ is Boltzmann's constant, $r_{s}$ is the electron spacing parameter--about unity in Jupiter and Saturn). For metallic hydrogen, this translates into a melting point $\mathrm{T}_{\mathrm{M}}$ of about $1500 \mathrm{p}^{1 / 3}$ $o_{\mathrm{K}}$, where $\rho$ is the density in $\mathrm{g} . \mathrm{cm}^{-3}$.

At low pressures, the metallic state is thermodynamically unfavorable relative to the molecular state. The molecular state is more difficult to calculate from first principles and is consequently not well understood, except at very low pressures $\left(P<10^{10} \mathrm{~Pa}\right.$ ) where experiments provide accurate information. The transition between molecular and metallic hydrogen is believed to occur at about $2 \times 10^{11} \mathrm{~Pa}$, based on the most recent analysis of isentropic data (Hawke et al. 1978), but large uncertainties remain in both the data and the interpretation. Any value in the range $1 \times 10^{11}-5 \times 10^{11} \mathrm{~Pa}$ is compatible with current knowledge (Stevenson and Salpeter, 1977a). The best current procedure for modelling molecular hydrogen (Ross, 1974 ) is to construct a pair potential compatible with known experimental data, even though the concept of a pair potential ceases to be strictly valid at P $10^{11} \mathrm{~Pa}$

Figure 2 shows a comparison between the expected temperature profiles (adiabats) in Jupiter and Saturn and the phase diagram for pure hydrogen. Although there are obviously large uncertainties at high pressures, it seems likely that the adiabats cross at most one phase boundary, the molecularmetallic transition. This establishes that Jupiter and Saturn have essentially bottomless atmospheres. Even the liquid molecular-liquid metallic transition may not be first oxder. This controversial issue is very important to the thermal and dynamic state of Jupiter and Saturn (Stevenson and Salpeter, 1977a and 1977b) but is not likely to be resolved until relevant

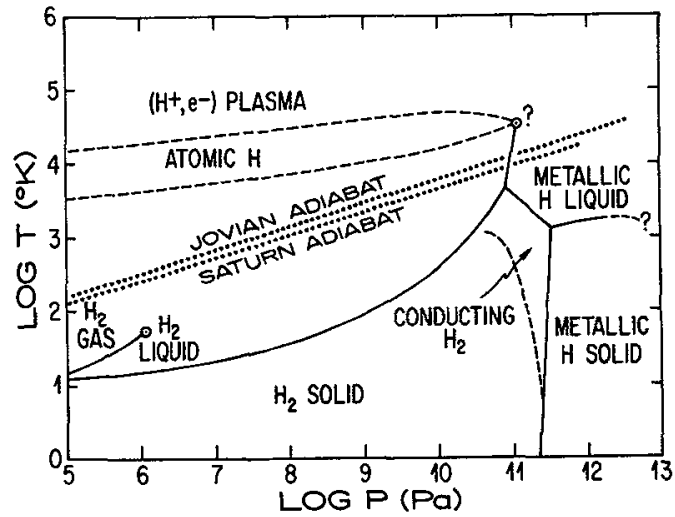

Fig. 2 : Hydrogen phase diagram and superimposed planetary adiabats.

experiments have been carried out. Figure 2 also shows that the adiabats do not cross the freezing curves of either molecular or metallic hydrogen. If there are no first order phase tranistions encountered along an adiabat, the the calculation of temperatures in the deep interiors of Jupitex and Saturn is straightforward (Hubbard, 1973). One simply equates the entropy per atom in the atmosphere to the entropy per atom in the metallic hydrogenhelium region. Both of these can be readily calculated, even though the entropy of intermediate high density molecular states cannot. In the metallic region, Monte Carlo calculations (Hubbard and slattery, 1971; Dewitt and Hubbard, 1976) and fluid perturbation theory (Stevenson, 1975) essentially agree. The resulting central temperatures are around $20,000 \mathrm{~K}^{\mathrm{K}}$ for Jupiter and $11,000^{\circ} \mathrm{K}$ for Saturn, the difference being due primarily to the lower central pressure in saturn but also due in part to the lower atmospheric temperature of saturn. These estimates will be incorrect, if the molecular-metallic phase transition is still first order at $10,000^{\circ} \mathrm{K}$ (Salpeter and Stevenson, 1976).

The main unresolved issues for pure hydrogen are :

(a) That is the behavior of molecular hydrogen around pn10IIPa? (It is probakly a semiconductor, at least.) (b) Where is the molecular-metallic transition and what is the critical temperature above which the transition ceases to be first order? (c) What are the electrical and thermal conductivities of liquid, metallic hydrogen? These are known to about a factor of three (Stevenson and Ashcroft, 1974 ; Stevenson and salpeter, 1977a) but better estimates would be useful. 3. Hydrogen-HeIium Iixtures.- There has been much less experimental work on helium than on hydrogen. Theoretical calculations (see stevenson and salpeter, 1977a, for a review) indicate that pure helium 
does not become metallic until a pressure of around $7 \times 10^{2} 2 \mathrm{~Pa}$, well in excess of the highest pressure in the hydrogen-helium region of either Jupiter or Saturn. It is more relevant, however, to consider the behavior of a hydrogen-helium mixture containing about $8-10 \%$ helium by atomic number (25-30\% by mass). The existence of a large amount of heat emanating from the interiors of Jupiter (about $4 \times 10^{24} \mathrm{erg} / \mathrm{sec}$ ) and Saturn (about $1.5-2 \times 10^{24} \mathrm{erg} / \mathrm{sec}$ ) prompted consideration of the possibility that helium might have limited solubility in hydrogen, since this would cause gravitational differentiation and energy release (Salpeter, 1973). Calculations on a fully-pressure ionized hydrogen-helium mixture (stevenson, 1975; Hansen and Viellefosse, 1976; Straus et al., 1977; Pollock and Alder, 1977; Firey and Ashcroft, 1977) confirmed the existence of a miscibility gap. It is now apparent that this is a ubiquitous property of fully-pressure ionized mixtures (Stevenson, 1976; Brami et al., 1979) and not a special property of hydrogen-helium. These calculations suggested that a liquid cosmic mixture would phase separate into hydrogen-rich and helium-rich liqui phases at a temperature of axound $8000-11,000^{\circ} \mathrm{K}_{i}$ almost independent of pressure. A remarkable feature of this result is that this temperature is about the same as that at the molecular-metallic transition in Jupiter and Saturn. This could be coincidence, or it could be that the onset of the phase separation releases so much enexgy that it prevents the planet from cooling beyoni that point, at least over the age of the solar systerl. In fact, the energy output of Jupiter can be entirely explained by the gradual loss of the primordial heat that was rapidly generated during the accretion and collapse accompanying the formation of the planet (Graboske et al., 1975). At the time this is written, the situation concerning saturn is less clear, but the ground-based observations suggest that the luminosity is higher than would be expected from građual cooling alone (Ṕllack et al., 1977). Phase separation of the helium may be required. The results from Pioneer 11 (which passed saturn on september 1 , 1979) and the Voyager spacecraft (1980, 1981) will help in assessing this possibility. If helium is undergoing differentiation then it should also be depleted from the atmosphere, but this is very difficult to detect by remote sensing.

A serious objection to helium differentiation is that the helium may not be fully pressure ionized in the presence of metallic hydrogen, if the pressure is only ${ }^{1} 10^{12} \mathrm{~Pa}$. Unfortunately, this pressure regime is very difficult to treat theoretically. In the low pressure limit, the repulsive pseudopotential of the helium atom immersed in an electron gas ensures a very low solubility (Stevenson, 1979a; also unpublished calculations by Pollock and Alder based on their 1977 paper). The results suggest (but do not prove) that the solubility of helium in metallic hydrogen increases monotonically with pressure. If this is so, then limited solubility is certainly important in the evolution of saturn and (eventually) Jupiter.

In Figure 3, planetary adiabats are compared with the temperature at which helium is saturated in hydrogen. Theorical calculations have also been made for the molecular mixture (Stevenson and Salpeter, 1977a) but the temperature for insolubility

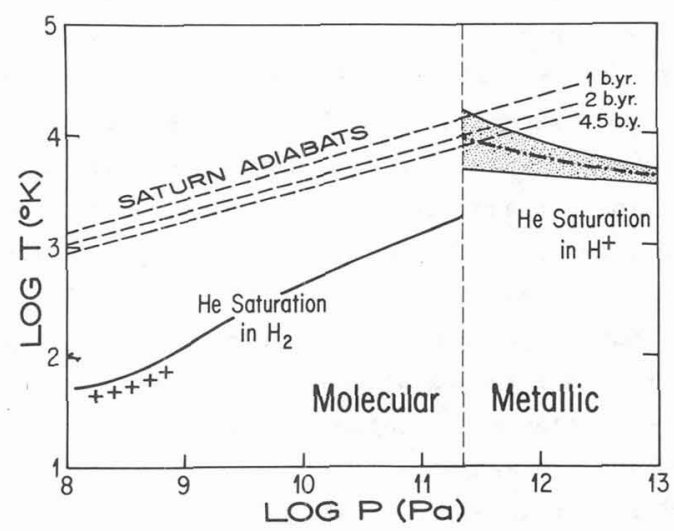

Fig. 3 : Helium saturation for a cosmic mixture and several saturn adiabats for different evolutionary ages. Shading represents theoretical uncertainly. Crosses are experiments (Streett, 1974).

is almost certainly too low to be important. The insolubility occurs first at the molecular-metallic transition, and would lead to the formation of helium raidrops. These can grow and fall, despite the convection that is present, leading to enexgy release and the eventual formation of a helium-rich core.

Another feature of metallic hydrogen-helium mixtures is a significant volume non-additivity. It is usually assumed by planetary modellers that the pressure-density relationship of a mixture can be found by assuming that the mixture volume is 
the sum of the volumes of the constituents at the same pressure (i.e. there is no volume change upon alloying). This is certainly not true for helium dissolved in metallic hydrogen (Stevenson, 1979a). The non-additivity increases the density for a given composition, so models of Jupiter and saturn require less helium than they would if volume additivity were correct. The consequence of this is that models of Jupiter and Saturn can be constructed invoking cosmic abundances of helium ( $25 \%$ by mass) whereas volume additivity would suggest $30-35 \%$ helium by mass, rather larger than the observed galactic value or the value predicted by the big bang. It is alternatively possible to increase the density by enhancing the rock or ice component above cosmic abundance. This may be needed to produce a qmal.l dense central region and explain the gravitational field $\left(\mathrm{J}_{2}, \mathrm{~J}_{4}\right)$ but it is not needed to explain the density in the metallic hyarogen region.

It is not possible in this short review to describe in detail the procedure for constructing models of Jupiter and Saturn. Interested readers should consult Stevenson (1978) or Zharkov and Trubitsyn (1978). In Figure 4, a schematic representation of a "typical"

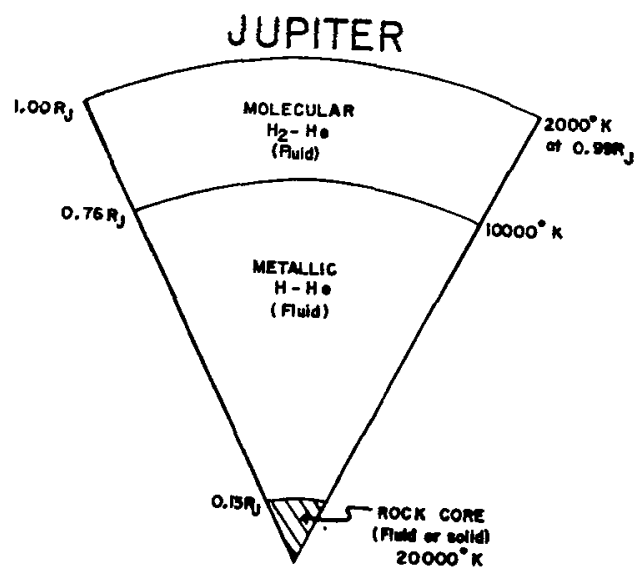

Fig.4.- A "Typical" Jupiter model.

Jupiter model (Stevenson and Salpeter, 1976) is shown. This particular model contains a small "rock" core (density ranging from 18 to $26 \mathrm{~g} \cdot \mathrm{cm}^{-3}$, pressure ranging from 4.5 to $10 \times 10^{12} \mathrm{~Pa}$ ) of around five earth masses. The highest pressure in the hydrogenhelium region is $\sim 4.5 \times 10^{12} \mathrm{~Pa}$, where the density is $24.3 \mathrm{~g} \cdot \mathrm{cm}^{-3}$ and the temperature is $20,000^{\circ} \mathrm{K}$. The outer boundary condition is $\mathrm{T}=160^{\circ} \mathrm{K}$ at $\mathrm{P}=10^{5} \mathrm{~Pa}$, and the total helium abundance is $\sim 26 \%$ by mass.

4. Icy bodies. - Uranus, Neptune, Pluto and a number of large satellites (most notably Ganymede, callisto, Titan) are rich in at least some of the "ices": water, methane and ammonia. This is not surprising since the cosmic abundance of oxygen by mass is an order of magnitude greater than that of magnesium or silicon, and almost an order of magnitude greater than that of iron. The abundance of carbon and nitrogen are also greater than those of the rockforming elements.

The high pressure behavior of the ices, and especially of water, is more complicated than the behavior of hydrogen or helium. Water is known to possess at least eight phases (seven solid, one liquid) not counting the inevitable metallic phase. The formation of metaliic $H_{2} O$ has been claimed in static experiments (Vereshchagin et al., 1975; Kawai et al., 1977) but the evidence is still unconvincing. It is known that water becomes pressuredissociated into $\mathrm{H}_{3} \mathrm{O}^{+} \mathrm{OH}^{-}$above about $1.5 \times 10 \mathrm{~Pa}$ (Hamann and Linton, 1966) and this almost cextainly explains why the equation of state deduced from recent shock data (Nellis, 1978) is softer than previous estimates. In Uranus and Neptune, the actual temperature (assuming, as usual, an adiabatic structure) should exceed the freezing point and it is necessary to understand the thermodynamics of ionic melt ( $\mathrm{H}_{3} \mathrm{O}^{+} \mathrm{OH}^{-}$) and how this alloys with other constituents such as $\mathrm{H}_{2}, \mathrm{NH}_{3}$ and $\mathrm{CH}_{4}$. This has not been done yet, so current models of Uranus and Neptune (e.g. Hubbard and MacFarlane, 1979) are sti11 "simple" in the sense that they make arbitrary assumptions about the layering of the planet. Model construction is also hindered by the large uncertainties in the observations, including such fundamental properties as the rotation rate (which is needed before one can estimate the moment of inertia from the gravitational moment $\mathrm{J}_{2}$ or the oblateness). One interesting feature of the atmospheres of Uranus and Neptune is that they appear to have methane enrichment (Owen and cess, 1975) yet a lower than cosmic abundance of ammonia (Gulkis et al., 1978). It is possible that this is a consequence of processes taking place deep within the planet. For example, $\mathrm{NH}^{3}$ and $\mathrm{H}_{2}$ may form an $\mathrm{NH}_{4}^{+} \mathrm{OH}^{-}$alloy at $\mathrm{P}>10^{1}-\mathrm{Pa}$, whereas $\mathrm{CH}_{4}$ (which has a low proton affinity) remains neutral and undifferentiated. The modification of atmospheric and surface chemistry by processes deep within a planet is a familiar notion to geochemists, but has yet 
to be seriously considered by those scientists who study the major planets.

In the icy satellites, where the atmosphere is usually thin (Titan being a remarkable exception), a different problem arises: How do solid ices flow at moderately high pressures $\left(\mathrm{P} \sim 10^{\circ} \mathrm{Pa}\right)$ and low temperatures? subsolidus convection, which is responsible for plate tectonics and continental dirft on earth (and, presumably, Venus) may also cause ice tectonics on the icy satellites (see, for example, Golitsyn, 1979).

5. Terrestrial bodies. - The discussion here will be confined to the behavior of iron cores. By an unfortunate accident of nature, iron, the most abundant heavy element in the universe (because of its very favorable nuclear structure), has a very complicated electronic structure (an incompletely filled 3d-band contained within a partially occupied $4 \mathrm{~s}$-band). However, band structure calculations (e.g. Bukowinski, 1976) indicate that an electronic structure close to $3 d^{7} 4 s^{1}$ persists in the metal at all pressures up until around $10^{2} \mathrm{~Pa}$, where a transition to $3 \mathrm{~d}^{8}$ occurs. This transition might be encountered near the center of Jupiter but is certainly not important for the interiors of the terrestrial planets. It is possible, therefore, that the low pressure behavior of iron provides some guidance for its behavior in the earth' $s$ core. In this respect, iron differs from the gases and ices which have very different low pressure and high pressure behaviors.

Despite this advantage, no realistic models have been made yet for the thermodynamics of liquid iron at core pressures. All existing discussions (see, for example, stacey, 1977) are based on solid-state considerations. The simple hard-sphere like behavior of the low pressure liquid structure factor of iron suggests that is should be possible to identify a pair potential Application of Monte Carlo techniques or Iiquid perturbation theory (guided, of course, by the high pressure data from shock or static experiments) may then lead to useful predictions concerning the thermodynamics and transport properties of liquid iron at $\mathrm{P}>10^{11} \mathrm{~Pa}$ (Stevenson, 1979b).

It has been known for many years from seismic data and shockwave data that the earth's outer liquid core is about ten percent less dense than pure iron or an ixon-nickel alloy at the same pressure and temperature (see Jacobs, 1975, for a review). This is best explained by the presence of a light alloying constituent. The most likely candidate is sulphur (Ahrens, 1979), although a significant amount of oxygen might be present, if some of the separation of iron from silicates occured at high pressures (Jeanloz and Ahrens, 1979). A much more exotic possibility is that the earth's core is an alloy of iron and metallic hydrogen (Stevenson, 1977).

6. Future Prospects. - In many respects, the interior of Jupiter is better understood than the interior of any other planet, including the earth. This is due, in part, to the relative simplicity of hydrogen. (It is also partly a consequence of the relative slow rate at which geophysicists adopt the most recent advances in condensed matter physics!). However, the study of planetary interiors has two major advantages over allied studies of the condensed mattex physics of white dwarfs or neutron stars; the possibility of relevant in situ measurements by spacecraft, and the possibility of relevant high pressure laboratory expeximents. It is these two factors which together will ensure future progress

in this subject. Even a mere descade from now, a lot of what is said in this paper may be either superseded or common knowledge. Further in the future, we can look forward to the probing of planetary interiors by neutrino beams and the exploration of planets in other solar systems.

\section{References}

Ahrens, T.J. (1979) Equations of state of iron sulfide and constraints on the sulfur content of the earth. J. Geophys. Res, 84, 985.

Birch, F. (1968) on the possibility of large changes in the earth's volume. Phys. Earth Planet. Int. 1,141 .

Brami, B., Hansen, J.P. and Joly, F (1979) phase separation in highly disymetric binary ionic mixtures. Physica 95A, 505.

Bukowinski, M.S.T. (1976) on the electronic structure of iron at core pressures. Phys. Earth Planet. Int. 13,57 .

Dewitt, H and Hubbard, W.B. (1976) statistical mechanics of light elements at high pressures. IV A model free energy for the metallic phase. Astrophys. J. 205, 295.

Firey, B. and Ashcroft, N.W. (1977) Thermodynamics of Thomas-Fermi screened Coulomb systems. Phys. Rev. A15, 2072

Golitsyn, G.S. (1979) Convection in the ice crusts of satellites and its effect on surface relief. Geophys. Rev. Lett. 6, 466.

Graboske, H.C. Jr., Pollack, M.B., Grossman, A.S. and Olness, R.J. (1975) The structure and evolution of Jupiter: The fluid contraction stage. Astrophys. J. $199,265$.

Gulkis, S. Janssen, M.A. and Olsen, E.T. (1978) Evidence for depletion of ammonia in the Uranus atmosphere. Icarus $34,10$. 
Hamann, S.D. and Linton, M. (1966) Electrical conductivity of water in shock compression. Trans. Faraday Society (GB)) $62,2234$.

Hansen, J-P, and Viellefosse, P. (1976) Equation of state of the classical two-component plasma. Phys. Rev. Lett $37,391$.

Fawke, P.S., Burgess, T.J., Duerre, D.E., Huebe1, J. G., Keeler, R.N., Klapper, H. and Wallace, W.C. (1978) Observations of electrical conductivity of isentropically compressed hydrogen at megabar pressures. Phys. Rev. Lett. 14, 994.

Hubbard, W.B. (1973) Observational constraint on the structure of hydrogen planets. Astrophys. J. 182, L35.

Hubbard, W.B. and Slattery, W.I. (1971) Statistical mechanics of light elements at high pressure. Astrophys. J. 168, 131 .

Hubbard, W.B. and MacFarlane, J.J. (1979) Structure and evolution of Uranus and Neptune. Submitted to J. Geophys. Res.

Jacobs, J.A. (1975) "The Earth's core" (Academic Press, N.Y.) $253 \mathrm{pp}$.

Jeanloz, R. and Ahrens, T.J. (1979) Equations of state of $\mathrm{FeO}$ and $\mathrm{CaO}$. Preprint.

Kawai, N., Togaya, M. and Mishima, O. (1977) Metallic transitions of oxides, $\mathrm{H}_{2} \mathrm{O}$ and hydrogen. In "High Pressure Research: Applications in Geophysics", ed. M.H. Manghnani and S.-I. Akimoto (Academic Press, N.Y.) p. 267.

Iyttieton, R.A. (1973) The end of the iron-core age. The Moon 7,422 .

Nellis, W.J. (1978) private communication (Lawrence Livermore Laboratory).

Owen, T. and Cess, R.D. (1975) Methane absorption in the vivible spectra of the outer planets and Titan. Astrophys. J. Lett. 197, I37

Pollack, J.B., Grossman, A.S., Moore, R. and Graboske, H.C. Jr. (1977) A calculation of Saturn's gravitational contraction history. Icaxus 30, 111.

Pollock, E.L. and Alder, B.J. (1977) Phase separation for a dense fluid mixture of nuclei. Phys. Rev. A15, 1263.

Pollock, E.I. and Hansen, J-P. (1973) Statistical mechanics of dense ionized matter. Phys. Rev. 8A, 3110 .

Ramsey, W.H. (1949) On the nature of the earth'core. Mon. Not. Roy. Astr. Soc. Geophys. Suppl. 5, 409.

Ross, M. (1976) A theoretical analysis of the shock compression experiments of the liquid hydrogen isotopes and a prediction of the metallic transition. J. Chem. Phys. 60, 3634.

Salpeter, E.E. (1973) on convection and gravitational layering in Jupiter and in stars of low mass, Astrophys. J. Lett. 181, L83.

Salpeter, E.D. and Stevenson, D.J. (1976) Heat transport in a stratified two-phase fluid. Phys. Fluids 19, 502 .

stacey, F.D. (1977) A thermal model of the earth. Phys. Earth Planet. Int. 15, 341.

Stevenson, D.J. (1975) Thermodynamics and phase separation of dense fully-ionized hydrogenhelium fluid mixtures. Phys. Rev. B12, 3999.

(1976) Miscibility gaps in fully pressureionized binary alloys. Phys. Lett. 58A, 282.
(1977) Hydrogen in the earth'core. Nature 268, 130.

(1978 The outer planets and their satellites. In "The origin of the solar system", ed. S.F. Dermott (J. Wiley \& Sons, N.Y.) p 395.

(1979a) Solubility of helium in metallic hyarogen. J. Phys. F: Metal Pysics 9, 791.

(1979b) Applications of liquid state physics to the earth's core. Submitted to Phys. Earth Planet. Int., in press.

Stevenson, D.J. and Salpeter, E.E. (1976) Interior models of Jupiter. In "Jupiter", ed. T. Gehrels (University of Arizona Press, Tucson). p. 85.

(1977a) The phase diagram and transport properties of hydrogen-helium fluid planets. Astrophys. J. Suppl. 35, 221.

(1977b) The dynamics and helium distribution in hyarogen-helium fluid planets. Astrophys. J. Suppl. 35, 239.

Straus, D.M., D.M., Ashcroft, N.W. and Beck, H. (1977) Phase separation of metallic hydrogenhelium alloys. Phys. Rev. B15, 1914.

Streett, W.B. (1974) Phase equilibria in molecular $\mathrm{H}_{2}$-He mixtures at high pressures. Astrophys. J. 186,1107 .

Vereshchagin, L.F., YakovIev, E.N. and Timofeev, Yu. A. (1975) Transition of $\mathrm{H}_{2} \mathrm{O}$ into the conducting state at static PrlMbar. J.E.T.P. Lett. 21,304 .

Zapolsky, H.S. and Salpeter, E.E. (1969) The massradius relationsiip for cold bodies of low mass. Astrophys. J. 158, 809.

Zharkov, V.N. and Trubitsyn, V.P. (1978) "Physics of Planetary Interiors", Pachart publ. (Tucson), $338 \mathrm{pp}$. 